\title{
A characterization of $F$-algebras with all one-sided ideals closed
}

\author{
by \\ W. Żelazko (Warszawa)
}

\begin{abstract}
We prove that a real or complex $F$-algebra has all left and right ideals closed if and only if it is noetherian.
\end{abstract}

A topological algebra is a real or complex algebra $A$ which is a topological vector space (t.v.s.) and the multiplication $(x, y) \mapsto x y$ is a jointly continuous map from $A^{2}$ to $A$. In terms of neighbourhoods of zero this means that for each such neighbourhood $U$ there is a neighbourhood $V$ with

$$
V^{2} \subset U \text {. }
$$

A unital topological algebra $A$ is called a $Q$-algebra if the set (group) $G(A)$ of all invertible elements of $A$ is open. It is known ([7, Lemma I.6.4, pp. 43-44]) that $A$ is a Q-algebra if and only if its unity element $e$ has a neighbourhood consisting of invertible elements.

An $F$-algebra is a topological algebra which is an $F$-space, i.e. a complete metrizable t.v.s. The topology of an $F$-space $X$ can be given by means of an $F$-norm, i.e. a map $x \mapsto\|x\|$ from $X$ to the non-negative real numbers such that

(i) $\|x\| \geq 0$ for all $x \in X$, and $\|x\|=0$ iff $x=0$,

(ii) $\|x+y\| \leq\|x\|+\|y\|, x, y \in X$,

(iii) the map $(\lambda, x) \mapsto\|\lambda x\|$ is jointly continuous, $\lambda \in \mathbb{K}(\mathbb{K}=\mathbb{R}$ or $\mathbb{C})$, $x \in X$.

For further information on $F$-spaces and $F$-norms the reader is referred to [1] and [8].

A topological algebra is said to be multiplicatively convex (briefly $m$ convex) if its topology can be given by means of a family of submultiplicative (algebra) seminorms. An m-convex algebra which is also an $F$-algebra is

2000 Mathematics Subject Classification: 46H10, 13E05. 
called an $m$-convex $B_{0}$-algebra. More information about topological algebras can be found in [7] or [10].

We say that an algebra $A$ is noetherian if it satisfies the ascending chain condition, i.e. whenever

$$
I_{1} \subseteq I_{2} \subseteq \cdots
$$

is a sequence of left (or right) ideals in $A$, then there is an index $n_{0}$ such that $I_{n}=I_{n_{0}}$ for all $n \geq n_{0}$. If $A$ is unital, then it is noetherian if and only if every proper one-sided ideal $I$ of $A$ is of the form

$$
I=x_{1} A+\cdots+x_{n} A, \quad x_{i} \in I,
$$

in the case of a right ideal, and

$$
I=A x_{1}+\cdots+A x_{n}, \quad x_{i} \in I,
$$

in the case of a left ideal.

The noetherian topological algebras were first considered by Grauert and Remmert in the context of commutative Banach algebras. They showed in [6] that a commutative noetherian Banach algebra is necessarily finitedimensional. This result was extended to the non-commutative case by Sinclair and Tullo [9]. Ferreira and Tomassini studied in [5] the noetherian m-convex algebras and showed, among other results, that a noetherian commutative complex unital m-convex $B_{0}$-algebra has all ideals closed (Theorem 2.6 of [5]). They also observed that there exist such infinite-dimensional algebras (the algebras of all power series in one or a finite number of variables).

Motivated by the work of Ferreira and Tomassini, Carboni and Larotonda ([2], [3]) constructed highly non-trivial examples of commutative semisimple noetherian m-convex $B_{0}$-algebras (the examples considered in [5] were radical algebras), which are principal ideal domains. Further, independently, Choukri and El Kinani in [4] and the author in [12] proved that a commutative $F$-algebra is noetherian if and only if it has all ideals closed. The aim of this paper is to extend this result to the non-commutative case (this solves a problem explicitly posed in [12]).

Our result reads as follows.

Theorem. Let $A$ be a real or complex F-algebra. Then $A$ has all onesided ideals closed if and only if it is noetherian.

In the proof we shall make use of the following result ([13, Corollary $])$.

Lemma 1. Let $A$ be a real or complex F-algebra with unity $e$.

(i) Let $\left(x_{n}\right)$ be a sequence of elements of $A$ tending to $e$. Then there is a subsequence $\left(a_{i}\right) \subset\left(x_{n}\right)$ such that for all natural $k$ the products

$$
u_{k}=\lim _{i} a_{k+i} a_{k+i-1} \cdots a_{k}
$$


and

$$
v_{k}=\lim _{i} a_{k} a_{k+1} \cdots a_{k+i}
$$

are convergent, with

$$
\lim _{k} u_{k}=\lim _{k} v_{k}=e .
$$

(ii) If $A$ is not a $Q$-algebra, then there is a sequence $\left(a_{i}\right)$ of non-invertible elements in $A$ tending to e such that either $I_{l}=\bigcup_{k} A v_{k}$ is a proper dense left ideal, or $I_{r}=\bigcup_{k} u_{k} A$ is a proper dense right ideal.

The easier part of our theorem follows from the following result, extending Lemma 2 of [12] to the non-commutative situation.

Lemma 2. Let $A$ be a real or complex F-algebra with all ideals closed. Then $A$ is noetherian.

Proof. Let $I$ be a proper right ideal in $A$ (for left ideals the reasoning is analogous) and choose a non-zero $x_{1} \in I$ so that $I_{1}=x_{1} A \subset I$. If $I=I_{1}$ we are done. If not, there is an $x_{2} \in I \backslash I_{1}$ and so $I_{2}=I_{1}+x_{2} A$ is a subideal of $I$. Again either $I_{2}=I$ and in this case we are done, or the process can be continued. If it does not finish, we obtain a sequence $I_{1} \subset I_{2} \subset \cdots \subset I$ of closed right ideals and all imbeddings are proper. Setting $J=\bigcup_{i \geq 1} I_{i}$ we obtain a proper right ideal in $A(J \subset I)$ which is not closed as the union of an increasing sequence of closed subspaces. This gives a contradiction showing that $I=I_{n}$ for some $n$, and the conclusion follows.

It remains to show that if $A$ is noetherian, then it has all left and right ideals closed. We obtain this result through Lemmas 3-7; first we need the concept of a topologically invertible element.

Definition. Let $A$ be a unital $F$-algebra. An element $u$ in $A$ is said to be topologically right (resp. left) invertible if there is a sequence $\left(z_{i}\right) \subset A$ with $\lim _{i} u z_{i}=e$ (resp. $\lim _{i} z_{i} u=e$ ). An element $u$ is said to be topologically invertible if it is both left and right topologically invertible. This concept can be extended to an arbitrary topological algebra $A$, but then sequences have to be replaced by nets in case $A$ is non-metrizable.

Let us recall the following simple lemma ([13, Lemma 1]); its proof follows immediately from the joint continuity of multiplication in $A$.

Lemma 3. Let $A$ be a real or complex F-algebra with unity $e$. Then for any $u, v \in A$ and $\varepsilon>0$ there is $a \delta=\delta(\varepsilon, u, v)>0$ such that

$$
\|x-e\|<\delta \quad \text { implies } \quad\|u x v-u v\|<\varepsilon
$$

for all $x$ in $A$. 
Taking in (5) a smaller positive $\varepsilon$, we can replace $\|x-e\|<\delta$ by $\|x-e\|$ $\leq \delta$, and in this form Lemma 3 will be used in the proof of Lemma 5 .

Lemma 4. A finite product of topologically left (resp. right) invertible elements is topologically left (resp. right) invertible.

Proof. First we show that the product of two topologically left invertible elements $a, b$ is again topologically invertible (for topologically right invertible elements the proof is the same). We have to show that for any $\varepsilon>0$ there is a $z \in A$ with

$$
\|z a b-e\|<\varepsilon .
$$

Since $b$ is topologically left invertible, there is a $z_{1} \in A$ such that

$$
\left\|z_{1} b-e\right\|<\varepsilon / 2 \text {. }
$$

By Lemma 3 (setting $u=z_{1}, v=b$ and replacing $\varepsilon$ by $\varepsilon / 2$ ), there is a $\delta>0$ such that

$$
\left\|z_{1} x b-z_{1} b\right\|<\varepsilon / 2
$$

whenever $\|x-e\|<\delta$. Finally, by the left topological invertibility of $a$, there is a $z_{2} \in A$ such that $\left\|z_{2} a-e\right\|<\delta$.

Consequently, setting in (8) $x=z_{2} a$, we obtain

$$
\left\|z_{1} z_{2} a b-z_{1} b\right\|<\varepsilon / 2
$$

which together with (7) gives (6). By an easy induction, any finite product of topologically left invertible elements is topologically left invertible. The conclusion follows.

In the proof of the following lemma we shall use a reasoning similar to the proof of Proposition 1 in [12] or Lemma 2 in [13].

Lemma 5. Let $A$ be a unital F-algebra, and $\left(x_{i}\right)$ a sequence of topologically left (resp. right) invertible elements tending to the unity e of $A$. Then there is a subsequence $\left(a_{i}\right) \subset\left(x_{i}\right)$ such that the products $u_{k}$ given by (2) (resp. $v_{k}$ given by (3)), $k=1,2, \ldots$, are topologically left (resp. right) invertible.

Proof. We assume that the $x_{i}$ are topologically left invertible. For topologically right invertible elements the proof is analogous. The sequence $\left(a_{i}\right)$ will be chosen inductively. First choose $x_{j_{1}}$ so that $\left\|e-x_{j_{1}}\right\|<1$, put $a_{1}=x_{j_{1}}$ and choose $z_{0,1}$ in $A$ so that $\left\|z_{0,1} a_{1}-e\right\|<1 / 2$; this is possible because $a_{1}$ is topologically left invertible. Using Lemma 3 , we choose a $\delta_{1}>0$ such that $\left\|z_{0,1} x a_{1}-z_{0,1} a_{1}\right\|<1 / 2$ whenever $\|x-e\| \leq \delta_{1}$. Put also $\delta_{0}=1$.

Suppose now that we have already chosen elements $a_{1}, \ldots, a_{n}, a_{i}=x_{j_{i}}$, $j_{i}<j_{i+1}$, elements $z_{i, k}$ in $A, 1 \leq k+i \leq n$, and positive numbers $\delta_{1}, \ldots, \delta_{n}$ 
in such a way that

$$
\left\|a_{k+i+1} a_{k+i} \cdots a_{k}-a_{k+i} \cdots a_{k}\right\|<2^{-(k+i+1)}
$$

$$
\text { for } 1 \leq k \leq k+i<n(i \geq 0),
$$

(10) $\left\|a_{k+i} \cdots a_{k}-e\right\|<\min \left\{\delta_{k-1}, k^{-1}\right\} \quad$ for $1 \leq k+i \leq n$,

(11) $\left\|z_{i, k} a_{k+i} \cdots a_{k}-e\right\|<\frac{1}{2(k+i)} \quad$ for $1 \leq k+i \leq n$,

$$
\left\|z_{i, k} x a_{k+i} \cdots a_{k}-z_{i, k} a_{k+i} \cdots a_{k}\right\|<\frac{1}{2(k+i)} \quad \text { for } 1 \leq k+i \leq n,
$$

provided

$$
\|x-e\| \leq \delta_{k+i}
$$

We shall show that it is possible to find elements $a_{n+1} \in\left(x_{i}\right), z_{i, n+1-i}$ in $A, 0 \leq i \leq n$, and a positive number $\delta_{n+1}$, so that (9)-(12) are satisfied with $n$ replaced by $n+1$. Let $\varepsilon>0$. Since $x_{i} \rightarrow e$, we can find an index $i(\varepsilon)$ such that

$$
\left\|a_{n} \cdots a_{k}-x_{i} a_{n} \cdots a_{k}\right\|<\varepsilon \quad \text { for } 1 \leq k \leq n,
$$

whenever $i \geq i(\varepsilon)$. There is also an index $j$ such that

$$
\left\|x_{i}-e\right\|<\min \left\{\delta_{n},(n+1)^{-1}\right\} \quad \text { whenever } i \geq j .
$$

Put

$$
s_{k}=\min \left\{\min \left\{\delta_{k-1}, k^{-1}\right\}-\left\|a_{k+i} \cdots a_{k}-e\right\|: 1 \leq k \leq k+i \leq n\right\} .
$$

By (10) all $s_{k}$ are positive, and hence so is $r=\min \left\{s_{k}: 1 \leq k \leq n\right\}$. Take now any $\varepsilon$ satisfying $0<\varepsilon<\min \left\{r, 2^{-(n+1)}\right\}$, put $i_{n+1}=\max \left\{i_{n}+1\right.$, $j, i(\varepsilon)\}$, and $a_{n+1}=x_{i_{n+1}}$. Relation (13) implies (9) with $n$ replaced by $n+1$, and also

$$
\left\|a_{n+1} a_{n} \cdots a_{k}-a_{n} \cdots a_{k}\right\|<r \leq s_{k} \leq \min \left\{\delta_{k-1}, k^{-1}\right\}-\left\|a_{n} \cdots a_{k}-e\right\|
$$

for $1 \leq k \leq n$, and so

$$
\begin{aligned}
\left\|a_{n+1} \cdots a_{k}-e\right\| & \leq\left\|a_{n+1} \cdots a_{k}-a_{n} \cdots a_{k}\right\|+\left\|a_{n} \cdots a_{k}-e\right\| \\
& <\min \left\{\delta_{k-1}, k^{-1}\right\} .
\end{aligned}
$$

Thus (10) holds true for $1 \leq k+1 \leq n+1$, provided $k \leq n$. The case $k=n+1$ is covered by (14), so that (10) is satisfied with $n$ replaced by $n+1$.

By the previous lemma the elements $a_{n+1} \cdots a_{k}$ are topologically left invertible, so there are $z_{i, k}, i+k=n+1$, such that relations (11) hold true with $n$ replaced by $n+1$. Finally, for the newly obtained elements $z_{i, n+1-i}$ we can find, by Lemma 3 , the desired $\delta_{n+1}>0$ so that (12) is satisfied. The induction step is complete. 
Relations (9) imply

$$
\begin{aligned}
\left\|a_{m+n} \cdots a_{k}-a_{n} \cdots a_{k}\right\| \leq & \left\|a_{m+n} \cdots a_{k}-a_{m+n-1} \cdots a_{k}\right\|+\cdots \\
& +\left\|a_{n+1} \cdots a_{k}-a_{n} \cdots a_{k}\right\| \\
\leq & 2^{-(m+n+1)}+\cdots+2^{-(n+1)}<2^{-n}
\end{aligned}
$$

for all natural $m, n$ and $k \leq n$. This shows the convergence of the products $u_{k}$ given by (2) for all natural $k$. Relations (10) imply

$$
\left\|u_{k+1}-e\right\| \leq \delta_{k}
$$

for all natural $k$. Fix such a $k$. By (11) and (12) we have

$$
\left\|z_{i, k} x a_{k+i} \cdots a_{k}-e\right\|<\frac{1}{k+i}
$$

for $\|x-e\| \leq \delta_{k+i}$. Setting now $x=u_{k+i+1}$ and taking into account the equality $u_{k}=u_{k+i+1} a_{k+i} \cdots a_{k}$, we obtain, by (15),

$$
\left\|z_{i, k} u_{k}-e\right\|<\frac{1}{k+i} \text {. }
$$

Letting $i \rightarrow \infty$, we see that $u_{k}$ is topologically left invertible. The conclusion follows.

We now pass to our crucial lemma.

Lemma 6. Let $A$ be a unital real or complex F-algebra which is noetherian. Then $A$ is a Q-algebra.

Proof. Assume that $A$ is not a Q-algebra. By Lemma 1(ii), there is a sequence $\left(a_{i}\right)$ of non-invertible elements in $A$ tending to the unity $e$ such that either $I_{r}=\bigcup_{k=1}^{\infty} u_{k} A$ is a proper dense right ideal, or $I_{l}=\bigcup_{k=1}^{\infty} A v_{k}$ is a proper dense left ideal. We can assume that $J=I_{r}$ is a proper ideal (the case of $I_{l}$ can be treated in an analogous way, or reduced to the former by considering $A$ with the reverse multiplication $x \circ y=y x$ ).

Since $A$ is noetherian, there are $y_{1}, \ldots, y_{n} \in J$ such that

$$
J=y_{1} A+\cdots+y_{n} A .
$$

Let $I_{k}=u_{k} A$, so that $I_{k}$ is a right ideal contained in $J$. Since $u_{k}=u_{k+1} a_{k}$ we have $I_{k}=u_{k+1} a_{k} A \subset I_{k+1}$ and $J=\bigcup_{k} I_{k}$. Thus there is the smallest index $k_{0}$ such that all elements $y_{1}, \ldots, y_{n}$ are in $I_{k_{0}}$. Consequently, $J=I_{k_{0}}=I_{k}$ for all $k \geq k_{0}$. Since $J$ is dense in $A$, for each $k \geq k_{0}$ there is a sequence $\left(z_{i}^{(k)}\right)_{i=1}^{\infty}$ of elements of $A$ such that

$$
\lim _{i} u_{k} z_{i}^{(k)}=e \quad \text { for all } k \geq k_{0} .
$$

This means that all elements $u_{k}, k \geq k_{0}$, are topologically right invertible.

We now show that they cannot be left invertible. Otherwise there are $c_{k} \in A$ such that $c_{k} u_{k}=e, k \geq k_{0}$, and (16) implies $c_{k}=\lim _{i} c_{k} u_{k} z_{i}^{(k)}=$ 
$\lim _{i} z_{i}^{(k)}$, so that $u_{k} c_{k}=\lim _{i} u_{k} z_{i}^{(k)}=e$. Thus all $u_{k}, k \geq k_{0}$, are invertible with inverses $c_{k}$. Writing $u_{k}=u_{k+1} a_{k}, k \geq k_{0}$, we obtain $a_{k}=u_{k+1}^{-1} u_{k}$, so that all $a_{k}, k \geq k_{0}$, are invertible, contrary to assumption. Consequently, the elements $u_{k}, k \geq k_{0}$, are not left invertible.

Setting $x_{i}=u_{k_{0}+i-1}$ we obtain a sequence of elements of $A$ which are topologically right invertible and not left invertible, and tend to $e$. By Lemma 5 there is a subsequence $\left(b_{k}\right) \subset\left(x_{i}\right)$ such that the elements $v_{k}$ given by (3) with $a_{i}$ replaced by $b_{i}$ are topologically right invertible and tend to $e$. A reasoning similar to the first part of the proof shows that the elements $v_{k}$ are not left invertible. Consequently, all $I_{k}^{\prime}=A v_{k}$ are proper left ideals, and so is $J^{\prime}=\bigcup_{k} A v_{k}$. Since $v_{k} \rightarrow e$, the ideal $J^{\prime}$ is dense. As before, we find an index $k_{1}$ such that $J^{\prime}=I_{k_{1}}^{\prime}=I_{k}^{\prime}$ for $k \geq k_{1}$. The relation $A v_{k}=A v_{k+1}$, $k \geq k_{1}$, implies $v_{k+1} \in A v_{k}$, and so there is a $d_{k} \in A$ with $v_{k+1}=d_{k} v_{k}$. But $v_{k}=b_{k} v_{k+1}$ and so $v_{k+1}=d_{k} b_{k} v_{k+1}$, or

$$
\left(e-d_{k} b_{k}\right) v_{k+1}=0 \text {. }
$$

Since the elements $v_{k}, k \geq k_{1}$, are topologically right invertible, there are sequences $\left(w_{i}^{(k)}\right)_{i=1}^{\infty}, k \geq k_{1}$, with $\lim _{i} v_{k} w_{i}^{(k)}=e$. Fixing a $k \geq k_{1}$, multiplying (17) from the right by $w_{i}^{(k+1)}$ and passing to the limit with respect to $i$, we obtain $d_{k} b_{k}=e$, i.e. $b_{k}$ is left invertible. This is the desired contradiction and the conclusion follows.

The main idea of the next lemma is due to Grauert and Remmert ([6, Chapter I, Remark 2 in the Appendix to §5]); they needed it to prove that a commutative noetherian Banach algebra is necessarily finite-dimensional. Here we give a modification of the proof of a similar lemma proved in [12] in the commutative case. Similarly to [12] we shall use neighbourhoods of the origin instead of $F$-norms.

Lemma 7. Let $A$ be a real or complex unital $F$-algebra which is also a Q-algebra. Let I be a proper left (resp. right) ideal in $A$ whose closure $\bar{I}$ is a finitely generated ideal. Then I is closed.

Proof. We shall give the proof for a right ideal. The other case is analogous. Since $A$ is a Q-algebra, $\bar{I}$ is a proper ideal in $A$ and, by assumption,

$$
\bar{I}=x_{1} A+\cdots+x_{n} A \quad \text { with } x_{1}, \ldots, x_{n} \in \bar{I} .
$$

Put

$$
\Phi\left(u_{1}, \ldots, u_{n}\right)=x_{1} u_{1}+\cdots+x_{n} u_{n} ;
$$

this is a continuous linear map from $A^{n}$ to $A$. Since $A^{n}$ is also an $F$-space (with the product topology), and $\Phi$ is onto, the Banach theorem (see [1] or [8]) says that $\Phi$ is open, and so, for every neighbourhood $V$ of zero in $A$, the set $S(V)=\Phi(V, \ldots, V)=x_{1} V+\cdots+x_{n} V$ is also a neighbourhood of zero 
in $\bar{I}$. Since $I$ is dense in $\bar{I}$ we have

$$
I+S(V)=\bar{I}
$$

for each such $V$. Since $x_{i} \in \bar{I}$, (18) implies that for any neighbourhood $V$ of zero in $A$ there are $u_{k, i}$ in $V$ and $y_{i}$ in $I, 1 \leq i, k \leq n$, such that $x_{k}=y_{k}+\sum_{i=1}^{n} x_{i} u_{k, i}$, or

$$
y_{k}=x_{k}-\sum_{i=1}^{n} x_{i} u_{k, i}, \quad k=1, \ldots, n .
$$

We can treat (19) as a system of linear equations with given $y_{k} \in I$ and $u_{k, i} \in V$, and by solving it for suitably chosen $V$, we show that the elements $x_{i}$ must belong to $I$.

Grauert and Remmert solved this system by the use of the classical Cramer formulas. Since we are in the non-commutative situation, and using determinants of non-commuting elements would be troublesome, we shall solve the system by induction with respect to the number of variables. By solving this system, for a suitable neighbourhood $V$, we shall show that the elements $x_{i}$ are in $I$, which implies $\bar{I} \subset I$, as required. In each step of the induction we shall modify the neighbourhood $V$. Let $n=1$ and denote by $V_{1}$ a neighbourhood of the origin in $A$ so that $e-V_{1} \subset G(A)$. Such a $V_{1}$ exists, since $A$ is a Q-algebra. The system (19) is now of the form

$$
y_{1}=x_{1}-x_{1} u_{1,1}
$$

with $y_{1} \in I, u_{1,1} \in V_{1}$ and $x_{1} \in \bar{I}$. Thus $y_{1}=x_{1}\left(e-u_{1,1}\right)$, so that $x_{1}=$ $y_{1}\left(e-u_{1,1}\right)^{-1}$ and $x_{1} \in I$ since $I$ is a right ideal and $e-u_{1,1}$ is invertible.

Suppose now that for a given natural $m$ and for all natural $k$ with $1 \leq$ $k<m$ there are neighbourhoods $V_{k}$ of zero in $A$, with $V_{j} \subset V_{j-1}$ for $1<j \leq k$, such that whenever $y_{1}, \ldots, y_{k} \in I$ and $u_{k, i} \in V_{k}$ for $1 \leq i \leq k$, then there are unique $x_{1}, \ldots, x_{k}$ satisfying (19), and these elements are in $I$. We are now looking for a suitable $V_{m} \subset V_{m-1}$ so that the above holds with $k=m$. Consider (19) with $n=m$ and take into account the last equation

$$
y_{m}=x_{m}\left(e-u_{m, m}\right)-\sum_{i=1}^{m-1} x_{i} u_{m, i} .
$$

Since we demand $V_{m} \subset V_{m-1} \subset V_{1}$, and $u_{m, m} \in V_{m}$, the element $e-u_{m, m}$ is invertible and relation (20) can be replaced by

$$
y_{m}\left(e-u_{m, m}\right)^{-1}=x_{m}-\sum_{i=1}^{m-1} x_{i} u_{m, i}\left(e-u_{m, m}\right)^{-1} .
$$

Hence

$$
x_{m}=y_{m}\left(e-u_{m, m}\right)^{-1}+\sum_{i=1}^{m-1} x_{i} u_{m, i}\left(e-u_{m, m}\right)^{-1}
$$


and substituting this to the first $m-1$ equations in (19) with $n=m$, we have

$$
\begin{aligned}
y_{k}= & x_{k}-\sum_{i=1}^{m-1} x_{i} u_{k, i}-\left[y_{m}\left(e-u_{m, m}\right)^{-1}\right. \\
& \left.+\sum_{i=1}^{m-1} x_{i} u_{m, i}\left(e-u_{m, m}\right)^{-1}\right] u_{m, m} \quad \text { for } k=1 \ldots, m-1,
\end{aligned}
$$

or

$$
\begin{aligned}
& y_{k}+y_{m}\left(e-u_{m, m}\right)^{-1} u_{m, m} \\
& =x_{k}-\sum_{i=1}^{m-1} x_{i}\left[u_{k, i}+u_{m, i}\left(e-u_{m, m}\right)^{-1} u_{m, m}\right] \quad \text { for } k=1 \ldots, m-1 .
\end{aligned}
$$

Clearly, $y_{k}+y_{m}\left(e-u_{m, m}\right)^{-1} u_{m, m}$ belongs to $I$ for each choice of $V_{m} \subset V_{1}$, so in order to make the induction step, we have to find $V_{m}$ so that

$$
u_{k, i}+u_{m, i}\left(e-u_{m, m}\right)^{-1} u_{m, m} \in V_{m-1}
$$

whenever $u_{k, i} \in V_{m}$. Note that the elements in (23) are the coefficients of $x_{1}, \ldots, x_{m-1}$ in $(22)$.

To this end first choose a neighbourhood $U_{1}$ of the origin so that $U_{1}+$ $U_{1} \subset V_{m-1}$, and a neighbourhood $U_{2}$ with $U_{2}+U_{2} \subset U_{1}$.

We now find a neighbourhood $U_{3}$ of zero so that both $U_{3}^{2}$ and $U_{3}^{3}$ are contained in $U_{2}$, and a neighbourhood $U_{4} \subset V_{1}$ so that $x \in e+U_{4}$ implies $(e-x)^{-1} \in e+U_{3}$. The possibility of choosing $U_{4}$ follows from the fact that $x \mapsto x^{-1}$ is continuous on Q-algebras of type $F$ (taking the inverse is continuous in an $F$-algebra if and only if the set $G(A)$ is a $G_{\delta}$-set, see [7] or $[10])$, so in particular it is continuous at $x=e$. We now put

$$
V_{m}=U_{1} \cap U_{2} \cap U_{3} \cap U_{4} .
$$

If all the $u_{m, i}, 1 \leq i \leq m$, are in $V_{m}$, then $\left(e-u_{m, m}\right)^{-1} \in U_{3}$, and so

$$
u_{m, i}\left(e-u_{m, m}\right)^{-1} u_{m, m} \in U_{3}\left(e+U_{3}\right) U_{3} \subset U_{3}^{2}+U_{3}^{3} \subset U_{2}+U_{2} \subset U_{1} .
$$

Consequently, the left hand side of (23) is in $U_{1}+U_{1} \subset V_{m-1}$ and we are done. By the inductive assumption, $x_{1}, \ldots, x_{m-1}$ are in $I$. Consequently, by (21), we also have $x_{m} \in I$, completing the induction step. Thus the conclusion follows.

Proof of the Theorem. Assume first that $A$ is unital. In view of Lemma 2, we have to show that if $A$ is noetherian, then its ideals are all closed. By Lemma 6, $A$ is a Q-algebra. Let now $I$ be a left ideal in $A$ (for right ideals the proof is analogous). Since $A$ is a Q-algebra, the closure $\bar{I}$ is a proper ideal, finitely generated by assumption. Lemma 7 now implies that $I$ is closed.

For a non-unital $F$-algebra $A$, denote by $A_{e}$ its unitization, i.e. the direct sum $\mathbb{K} \oplus A$, where $\mathbb{K}=\mathbb{C}$ or $\mathbb{R}$, with coordinatewise addition and scalar 
multiplication, and with the multiplication given by $(\lambda e+a)(\mu e+b)=$ $\lambda \mu e+\lambda b+\mu a+a b$, where $\lambda e+b=(\lambda, b)$. It is a unital $F$-algebra with $F$-norm $\|\lambda e+a\|=|\lambda|+\|a\|$ and with the unity $e$. Clearly $A_{e}$ contains $A$ as a closed two-sided ideal. The proof in the non-unital case now follows from the fact that $A$ is noetherian if and only if $A_{e}$ is, and from the following proposition.

Proposition. Let $A$ be a real or complex non-unital algebra of type $F$. Then $A$ has all one-sided ideals closed if and only if the same holds for its unitization $A_{e}$.

Proof. We give the proof for left ideals. (For right ideals it is analogous.) Observe first that if $I$ is a left ideal in $A$, then it is also a left ideal in $A_{e}$ (we have $\left.A_{e} I=(\mathbb{K} e+A) I \subset I+A I \subset I\right)$. Thus if all left ideals are closed in $A_{e}$, they are also closed in $A$. Conversely, suppose that all left ideals are closed in $A$ and let $J$ be a proper left ideal in $A_{e}$. Put $I=J \cap A$. This is a left ideal in $A$, for if $x \in A$ and $y \in I$, then $x y$ is both in $J$ and in $A$, so it is in $I$. We are already done if $J \subset A$, so assume there is an element $u_{0}$ in $J$ of the form $u_{0}=e+a_{0}, a_{0} \in A$. We cannot have $a_{0} \in I$, since otherwise $e \in J$ and $J$ is not proper. Let now $u$ be an arbitrary element in $J$, so $u=\lambda e+a$ with $a \in A$. We claim that $u=\lambda u_{0}+b$ with $b \in A$. In fact,

$$
u-\lambda u_{0}=a-\lambda a_{0},
$$

so this difference is both in $A$ and in $J$ and hence in $I$. Denoting it by $b$, we obtain $u=\lambda u_{0}+b$. Thus, $J=\mathbb{K} \oplus I$. This implies that $J$ is closed. For if $\lambda_{n} u_{0}+b_{n} \rightarrow v, v \in A_{e}$, then the sequence of scalars $\left(\lambda_{n}\right)$ is bounded. Otherwise, passing to a subsequence if necessary, we can assume $\lambda_{n} \rightarrow \infty$, so that $u_{0}+b_{n} / \lambda_{n} \rightarrow 0$ and $u_{0} \in I$, since $b_{n} \in I$ and $I$ is closed. This gives a contradiction, and so, passing again to a subsequence, we can assume that $\left(\lambda_{n}\right)$ is convergent, say to $\lambda_{0}$. Consequently, $a_{n} \rightarrow v-\lambda_{0} u_{0}$, so that $v-\lambda_{0} u_{0} \in I$ and hence $v$ is in $J$. Thus $J$ is closed and the conclusion follows.

It was shown in [12, Example 6] that there exists a complete commutative locally convex non-metrizable algebra which has all ideals closed, but is not noetherian. One can also give an easy example of such a non-commutative algebra. To this end consider a real or complex free algebra $A$ in countably many variables $t_{1}, t_{2}, \ldots$, provided with the maximal locally convex topology $\tau_{\max }^{\mathrm{LC}}$ (it is given by all seminorms on $A$, and its linear subspaces are all closed). It is shown in [11] that $A$ is a topological algebra. Clearly all onesided ideals in $A$ are closed, but it is non-noetherian. To see this, let $I$ be the linear span of all monomials of degree at least two. It is a two-sided ideal in $A$ and it is neither left nor right finitely generated. For, if $x_{1}, \ldots, x_{n}$ were such generators, then they would contain only finitely many variables, say $t_{1}, \ldots, t_{k}$, and each element of $I$ would contain at least one of those 
variables. But $t_{k+1}^{2}$ is in $I$ and does not contain any of those variables and so our claim follows.

Thus our result does not extend to the non-metrizable case. However, we do not know whether there is a complete topological algebra which is noetherian but has a non-closed one-sided ideal; the answer is not known even in the commutative case (in [12] we gave an example of an incomplete normed noetherian commutative algebra which has non-closed ideals).

We do not know either whether there exists an $F$-algebra with all left ideals closed but with some right ideals non-closed $\left(^{1}\right)$. Nor do we know whether left noetherian $F$-algebras coincide with such algebras with all left ideals closed (our Lemma 2 says, in fact, that if all left ideals of an $F$-algebra $A$ are closed then $A$ is left noetherian, but we do not know whether the converse is true).

\section{References}

[1] S. Banach, Théorie des Opérations Linéaires, Warszawa, 1932.

[2] G. Carboni and A. Larotonda, An example of a Fréchet algebra which is a principal ideal domain, Studia Math. 138 (2000), 265-275.

[3] - , Fréchet m-convex principal ideal domains, Comm. Math. 52 (2002), 190-208.

[4] R. Choukri and A. El Kinani, Topological algebras with ascending and descending chain conditions, Arch. Math. (Basel) 72 (1999), 438-443.

[5] A. V. Ferreira and G. Tomassini, Finiteness properties of topological algebras, Ann. Scuola Norm. Sup. Pisa 5 (1978), 471-488.

[6] H. Grauert and R. Remmert, Analytische Stellenalgebren, Springer, 1971.

[7] A. Mallios, Topological Algebras. Selected Topics, North-Holland, 1986.

[8] S. Rolewicz, Metric Linear Spaces, PWN, 1972.

[9] A. M. Sinclair and A. W. Tullo, Noetherian Banach algebras are finite dimensional, Math. Ann. 211 (1974), 151-153.

[10] W. Żelazko, Selected Topics in Topological Algebras, Aarhus Univ. Lecture Notes 31, 1971.

[11] - On topologization of countably generated algebras, Studia Math. 112 (1994), $83-88$.

[12] - A characterization of commutative Fréchet algebras with all ideals closed, ibid. 138 (2000), 293-300.

[13] -, A characterization of Q-algebras of type F, ibid. 165 (2004), 73-79.

Institute of Mathematics

Polish Academy of Sciences

P.O. Box 21, 00-956 Warszawa, Poland

E-mail: zelazko@impan.gov.pl

Received March 2, 2004

$\left({ }^{1}\right)$ Added in proof: The author has constructed such an $m$-convex $B_{0}$-algebra. The construction will be published elsewhere. 